\title{
Supplementation with Matured Hop Bitter Acids Improves Cognitive Performance and Mood State in Healthy Older Adults with Subjective Cognitive Decline
}

\author{
Takafumi Fukuda ${ }^{\mathrm{a}, *}$, Tohru Ohnuma ${ }^{\mathrm{b}}$, Kuniaki Obara $^{\mathrm{a}}$, Sumio Kondo ${ }^{\mathrm{c}}$, \\ Heii Arai ${ }^{\mathrm{b}}$ and Yasuhisa Ano $^{\mathrm{a}}$ \\ ${ }^{a}$ KIRIN Central Research Institute, Kirin Holdings Company, Ltd., Kanagawa, Japan \\ ${ }^{\mathrm{b}}$ Department of Psychiatry, Juntendo University Faculty of Medicine, Tokyo, Japan \\ ${ }^{\mathrm{c}}$ Fukushima Healthcare Center, Osaka, Japan
}

Accepted 23 April 2020

\begin{abstract}
.
Background: Prevention of age-related cognitive decline and depression is becoming urgent because of rapid growing aging populations. Effects of vagal nerve activation on brain function by food ingredients are inadequately investigated; matured hop bitter acid (MHBA) administration reportedly improves cognitive function and depression via vagal nerve activation in model mice.

Objective: We investigated the effects of MHBA supplementation on cognitive function and mood state in healthy older adults with perceived subjective cognitive decline.

Methods: Using a randomized double-blind placebo-controlled trial design, 100 subjects (aged 45-69 years) were randomly assigned into placebo $(n=50)$ and MHBA $(n=50)$ groups, and received placebo or MHBA capsules daily for 12 weeks.

Results: Symbol Digit Modalities Test (SDMT) score assessing divided attention at week 12 was significantly higher $(p=0.045)$ and $\beta$-endorphin at week 12 was significantly lower $(p=0.043)$ in the subjects receiving MHBA. Transthyretin in serum, a putative mild cognitive impairment marker, was significantly higher at week 12 in the MHBA group than in the placebo group $(p=0.048)$. Subgroup analysis classified by the subjective cognitive decline questionnaire revealed that in addition to improved SDMT scores, memory retrieval assessed using the standard verbal paired-associate learning tests and the Ray Verbal Learning Test at week 12 had significantly improved in the subgroup with perceived subjective cognitive decline and without requirement for medical assistance in the MHBA group compared with that in the placebo group.
\end{abstract}

Conclusion: This study suggested that MHBA intake improves cognitive function, attention, and mood state in older adults.

Keywords: Attention, dietary supplements, hops, subjective cognitive decline

\footnotetext{
${ }^{*}$ Correspondence to: Takafumi Fukuda, KIRIN Central Research Institute, Kirin Holdings Company, Ltd., Kanagawa, Japan. Tel.: $+81 \quad 80 \quad 1930$ 9968; E-mail: Takafumi_Fukuda@kirin.co.jp.
}

\section{INTRODUCTION}

Dementia and cognitive decline are growing global concerns in today's aging societies; however, there is a lack of promising drugs for their treatment. There is an increased focus on maintaining cognitive function in old age and delaying the onset of 
neurodegenerative diseases such as Alzheimer's disease (AD) [1]. Recent studies have reported that early interventions in older people with subjective cognitive decline (SCD) in the absence of objective neuropsychological dysfunction are effective in preventing cognitive decline [2]. In the past two decades, methods for improving brain function via vagus nerve stimulation (VNS) have been developed. VNS is a novel treatment method for cognitive disorders [3, 4] and a Food and Drug Administration approved treatment for drug-resistant epilepsy and depression [5]. These VNS therapies require surgical implantation of a small device that provides electrical stimulation to the vagus nerve, thus, they have limited non-medical application. Recent studies suggest that a practical and non-invasive nutritional approach to vagal nerve stimulation can improve brain function.

Epidemiological studies indicate that moderate consumption of alcoholic beverages is beneficial to cognitive function [6, 7]. Resveratrol, a polyphenol present in red wine, has been reported to improve cognitive function in animal models as well as humans [8].

Previous studies demonstrate that iso- $\alpha$-acids (IAAs), which are hop-derived bitter compounds commonly used to make beer, activate the vagal nerve system, resulting in the improvement of cognitive function and depression in the model mice [9, 10]. Additionally, mature hop bitter acids (MHBA), which have a chemical structure similar to that of iso- $\alpha$-acids, act within the intestines to stimulate the vagus nerve and increase norepinephrine secretion in the brain, resulting in the improvement of cognitive function and depression in the model animals [11-14]. These reports suggest that activation of the vagal nerve by food ingredients can improve cognitive function and psychological state. A preliminary experiment by our research group demonstrated that MHBA supplementation ( $35 \mathrm{mg} /$ day) improved subjects' scores on the verbal fluency test and the Stroop test (color-word interference), which evaluated memory and attention in healthy middle-aged adults (45-64 years old) [15].

We conducted the present study in order to evaluate the effects of MHBA on human cognitive function and mood state in older adults with SCD. We used neuropsychological tests, questionnaires, and objective mental stress markers to assess cognitive function and mood states. We also measured putative mild cognitive impairment (MCI) markers in serum [16]. Furthermore, to evaluate the association of the level of SCD and the effectiveness of the MHBA supplement, the subjects were characterized by an SCD questionnaire (SCD-Q) $[17,18]$ and subgroup exploratory analyses were performed.

\section{MATERIALS AND METHODS}

\section{Participants}

Our study subjects were native Japanese participants. The following criteria were assessed during the screening session by questionnaire and clinical examination. The inclusion criteria were: 1) aged 45-69 years; 2) primary language Japanese; 3) participants with perceived SCD judged by the SCD-Q [17], that is, those who answered "YES" to at least one of the 24 questions. The exclusion criteria were: 1) visual or hearing impairment; 2) suspected dementia based on Mini-Mental State Examination (MMSE) score $<24 ; 3$ ) history of cranial nerve disease; 4) current hormonal treatment; 5) irregular lifestyle; 6) habitual consumption of beer-based drinks (including non-alcoholic drinks); 7) high habitual consumption of alcohol (>20 g/day); 8) smoking habit; 9) participation in the same neuropsychological tests within the past year; 10) regular consumption of drugs affecting cognitive function; 11) history of clinical sleep-related disorders; 12) blood donation within the past three months; 13) participation in any other clinical trials; 14) pregnant or breastfeeding; 15) allergy to the test food; 16) diagnosed dry mouth; 17) diagnosed arrhythmia.

\section{MHBA and placebo supplements}

MHBA were prepared as described previously $[14,19]$. The same MHBA test samples were used in a previous clinical study [15]. MHBA were composed of 4'-hydroxyallohumulinones [13], 4'-hydroxyalloisohumulones [11], tricyclooxyisohumulones A [20], hulupones, and humulinones [13], which are oxidants of $\alpha$ and $\beta$-acids. Test capsules containing $35 \mathrm{mg}$ of MHBA or $35 \mathrm{mg}$ of dextrin (placebo) were prepared by Kirin Holdings Co. Ltd (Tokyo, Japan). The nutritional composition of the test capsules are presented in Table 1. We confirmed that there were no discernible differences between the appearance and taste of the MHBA and placebo capsules. Test subjects ingested the capsules orally with water every day for twelve weeks. Compliance was monitored by counting the number of capsules remaining at the end of the experiment. 
Table 1

Nutritional composition of matured hop bitter acids (MHBA) and placebo capsules (amount per dose/day)

\begin{tabular}{lcc}
\hline & Placebo capsule & MHBA capsule \\
\hline MHBA (mg) & 0 & 35 \\
Moisture (mg) & 73 & 61 \\
Protein (g) & 0.2 & 0.3 \\
Carbohydrate (g) & 0.6 & 0.5 \\
Energy (kcal) & 3.4 & 3.5 \\
Sodium (mg) & 0.07 & 0.12 \\
\hline
\end{tabular}

\section{Trial design}

This was a randomized double-blind placebocontrolled parallel-group comparative study carried out over twelve weeks in Osaka, Japan. Figure 1 shows the screening procedures we employed to find 100 participants. Since this exploratory study was the first clinical trial to evaluate the potential of MHBA for cognitive function, an appropriate sample size was calculated based on previous human trials evaluating the effects of food ingredients on cognitive function [21-23]. Primary screening included questionnaires for inclusion/exclusion criteria, MMSE, and clinical examination for safety assessments. Secondary screening included neuropsychological tests, autonomic nerve measurement, and subjective psychological assessments. Individuals who fulfilled the eligibility criteria were recruited and randomly allocated to either the MHBA or placebo group in a 1:1

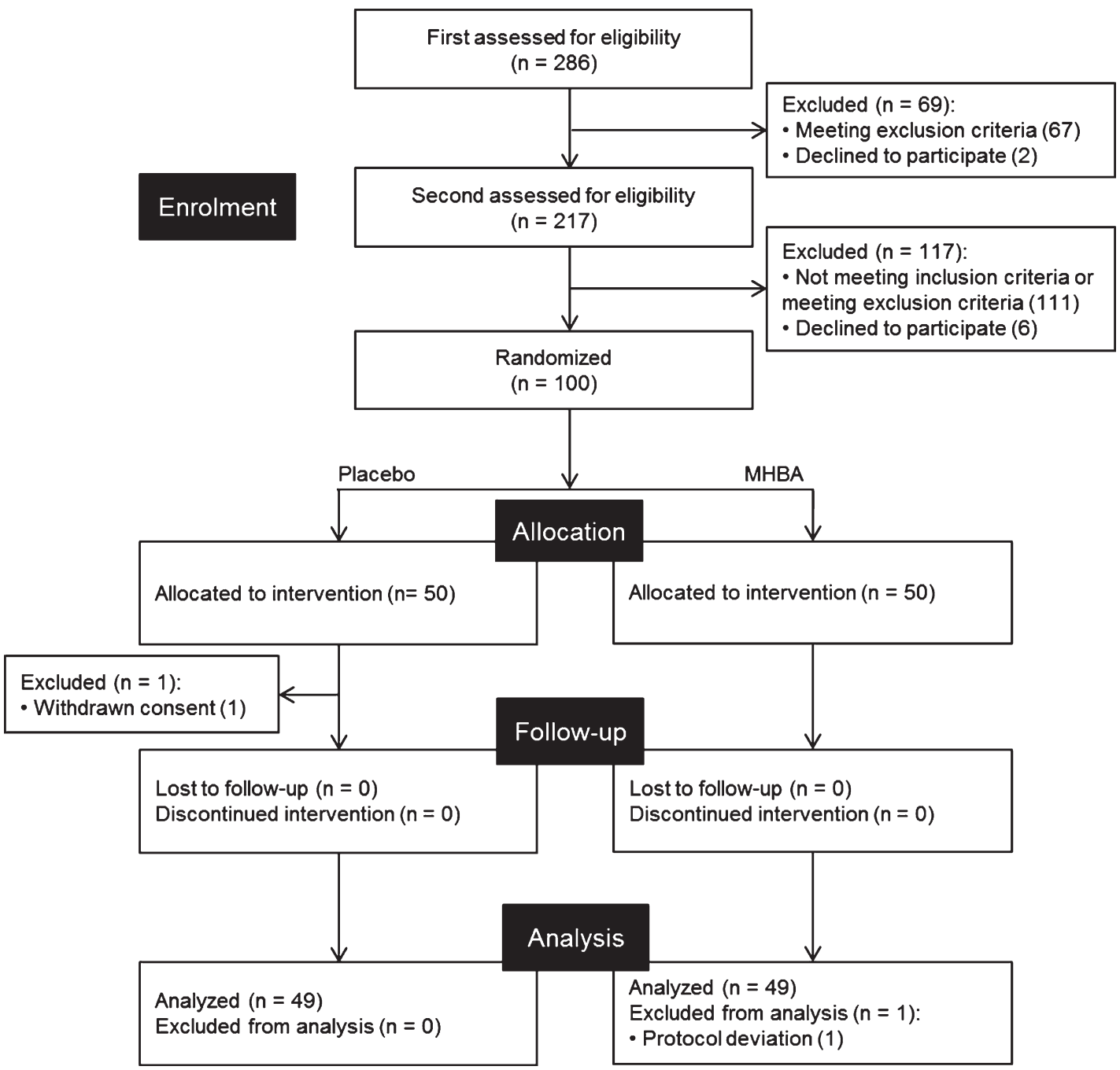

Fig. 1. CONSORT diagram. Out of the 286 subjects who were screened, 100 subjects were included in this study. These subjects were randomly allocated to placebo $(n=50)$ and MHBA $(n=50)$ groups. One subject in the placebo group withdraw consent and one subject in the MHBA group was excluded from analysis, leaving 49 subjects for analysis in each group. 
ratio by a third-party allocator using a computer program. The allocator was not involved in determining eligibility, data collection, or analysis. Research staff, outcome assessors and participants were blinded to the group allocation until the data analysis was complete.

Outcome assessments were performed at the baseline (week zero) and week twelve of the experiment. Participants were instructed to maintain their regular lifestyle, including eating habits, and to avoid using drugs or supplements that could affect cognitive function.

Neuropsychological tests were conducted under rigorously controlled conditions. In brief, on the day of the neuropsychological test, participants were instructed to abstain from consuming food and beverages other than water for $4 \mathrm{~h}$ before the tests. Data were collected at the DRC Co., Ltd. (Osaka, Japan), and the study was performed by the contract research organization TTC Co., Ltd. (Tokyo, Japan).

\section{Neuropsychological tests}

Neuropsychological tests to evaluate attention and memory were conducted by trained assessors who were blinded to the group allocation.

Attention was evaluated using the Clinical Assessment for Attention (CAT) [24]. The CAT consists of four tests. The Symbol Digit Modalities Test (SDMT) is used to assess divided attention. The SDMT is a cognitive test that consists of a sheet of paper with an answer key at the top (nine symbols and their correspondent numbers from 1 to 9) and a series of 120 random repetitions of those symbols [24]. The subject is required to enter the numbers that correspond to each symbol within a time limit of $90 \mathrm{~s}$. The cancellation task is used to assess selective attention and requires subjects to delete targets (sign 1, sign 2, number "3", and Japanese "ka") as fast as possible in interference stimulus. The memory updating test, in which participants were required to repeat the last three or four spans to the assessor was performed to assess working memory. The Position response test, in which Japanese words for "upper", "middle", or "lower" were printed in random positions, required participants to identify the position of the word instead of the meaning of the word.

Memory retrieval was evaluated using the Rey Auditory Verbal Learning Test (RAVLT) and S-PA. The word recall test was conducted according to the RAVLT [25]. Participants were presented with 15 words (List A) and asked to verbally recall them immediately and again after $5 \mathrm{~min}$ (total immediate memory (TIM); scores range from 0 to 75). Next, 15 different words (List B) were presented and participants were asked to verbally recall them as an interference task. Following the interference task, participants were asked to verbally recall List A immediately and then again after $20 \mathrm{~min}$ (T7). The number of forgotten words (T7-T6) was also evaluated. The verbal and visual paired-associate learning test was conducted according to the S-PA [26], in which subjects were presented pairs of words that were semantically unrelated. Subsequently, subjects were presented with one of each pair of words and were required to correctly recall the other. The trials were conducted three times and the total score was evaluated in the S-PA range of 0-30. A figural memory test was conducted according to the Wechsler Memory Scale-Revised (WMS-R) [27], in which subjects were required to memorize meaningless shapes. Subsequently, subjects were presented some meaningless shapes and were required to choose the memorized one.

\section{Mental stress markers in saliva}

To measure stress levels, saliva was collected at the baseline (week zero) and week twelve of the experiment, before and after the neuropsychological tests using salivette tubes (Sarstedt, Tokyo, Japan). Salivary $\beta$-endorphin, cortisol, chromogranin A $(\mathrm{CgA})$ and $\alpha$-amylase were assayed using commercially available kits ( $\beta$-Endorphine S-1134 kit, Peninsula Laboratories Inc., Belmont, CA, USA; Cortisol, EIA Kit, Salimetrics Inc., State College, PA, USA; Chromogranin A EIA kit, Yanaihara Institute, Shizuoka, Japan; $\alpha$-Amylase Assay Kit, Salimetrics Inc., State College, PA, USA).

\section{Mental stress, mood state, and subjective cognitive function assessments}

Anxiety traits that reflect long-term anxiety were evaluated prior to neuropsychological testing using the State-Trait Anxiety Inventory (STAI)-FormX-2 (scores range from 20 to 80) [28]. Subjective cognitive function was evaluated using the Metamemory in Adulthood Questionnaire (MIQ-A) which consists of six sub-categories; Change, Task, Capacity, Anxiety, Strategy, and Locus [29]. We also evaluated the change of subjective mood state during the neuropsychological tests using STAI-FormX-1 because anxiety-state may affect the neuropsychological test 
performance [30-32]. Subjective drowsiness was evaluated before and after the neuropsychological tests using the Karolinska Sleepiness Scale-Japanese version (KSS-J) [33].

\section{Indicators in serum}

Blood samples were collected after neuropsychological testing. Transthyretin (TTR), Apolipoprotein AI (ApoA1), and Complement 3 (C3) in serum were assayed using commercially available kits $(\mathrm{N}$ assay, TIA preALB, TIA ApoA1, and TIA C3-SH, Nittobo Medical Inc., Fukushima, Japan) as markers of MCI. [16]. Brain-derived neurotrophic factor (BDNF) was quantified using Human/Mouse BDNF DuoSet ELISA (R \& D Systems, Inc., Minneapolis, MN, USA). Cholecystokinin (CCK) was quantified using Human/Mouse/Rat CCK Enzyme Immunoassay Kit (RayBiotech, Peachtree Corners, GA, USA). These two indicators were evaluated to elucidate the underlying mechanism exploratory.

\section{A posteriori subgroup analysis}

Early diagnosis and treatments for the prevention of dementia are gathering increased attention. In particular, adults with perceived SCD are regarded as important candidates for early intervention. Previous research indicates that SCD can be divided into two groups: Those with perceived subjective cognitive decline (SDC-P) who have not sought medical assistance for their complaints, and those with clinical subjective cognitive decline (SCD-C) who have sought medical assistance [34]. Our exploratory study focused on these two SCD groups and subgroup analysis was performed to evaluate the effects of MHBA supplementation on cognitive function. Subjects were classified by their answers to the SCD-Q questions: 1) "Do you perceive memory or cognitive difficulties?", 2) "Would you ask a doctor about these difficulties?", and 3) "In the last two years, has your cognition, or memory declined?" $[17,18]$. Subjects who answered "YES" to all three questions were categorized as SCD-C and subjects who answered "YES" to the first and third questions and "NO" to the second question were categorized as SCD-P.

\section{Statistical analysis}

Data analysis was performed by a member of the research staff blinded according to a predefined plan. The results were expressed as a mean with standard deviation. Statistical comparisons were performed using IBM SPSS Statistics 23 (IBM, New York, NY, USA) and BellCurve for Excel (Social Survey Research Information Co., Ltd., Tokyo, Japan). Comparisons of all results, except MIA-Q, KSS-J, and STAI, were conducted using paired (baseline and week twelve scores) or unpaired (between-group differences in mean week twelve scores and changes in score from the baseline) $t$ tests. The MIA-Q, KSS$\mathrm{J}$, and STAI results were analyzed using Wilcoxon signed-rank test (baseline and week twelve scores) or Mann-Whitney $U$ tests (between-group differences in mean week twelve scores and changes in score from the baseline). All $p$-values $<0.05$ were considered statistically significant. Furthermore, preliminary multiple comparisons were performed on neuropsychological tests using z scores. Statistical analysis predefined in the protocol was conducted by the contract research organization TTC Co., Ltd. (Tokyo, Japan) and the funder was not involved in the statistical analysis.

\section{Standard protocol approvals, registrations, and patient consents}

The study was conducted in accordance with the Declaration of Helsinki and Ethical Guidelines for Medical and Health Research Involving Human Subjects and was approved by the ethics committee of Kensho-kai (Osaka, Japan). Written informed consent was obtained from all participants. The study was registered on January 21, 2019 in the database of the University Hospital Medical Information Network (UMIN) prior to subject enrollment (Registration No. UMIN000035601).

\section{RESULTS}

\section{Subject characteristics}

Participants were screened between January and April 2019 and the experiment was conducted from April to July 2019. Out of the 286 candidates from the volunteer bank, a total of 100 participants were recruited; one participant withdrew consent during the trial. Thus, 99 participants completed the twelveweek study (Fig. 1). One participant was excluded from the analysis according to the exclusion criterion: regular consumption of drugs affecting cognitive function during the trial. Therefore, a total of 98 participants' results were analyzed and their characteristics are presented in Table 2. There were 
Table 2

Characteristics of the study participants at baseline

\begin{tabular}{lccc}
\hline Characteristics & $\begin{array}{c}\text { Placebo } \\
(n=49)\end{array}$ & $\begin{array}{c}\text { MHBA } \\
(n=49)\end{array}$ & $p$ \\
\hline Age & $53.3 \pm 4.9$ & $54.6 \pm 6.3$ & 0.25 \\
Male/female & $21 / 28$ & $20 / 29$ & 1.00 \\
MMSE score (/30) & $28.5 \pm 1.2$ & $28.3 \pm 1.2$ & 0.55 \\
SCD-Q a) (YES/NO) & $46 / 3$ & $40 / 9$ & 0.12 \\
SCD-Q b (YES/NO) & $18 / 31$ & $16 / 33$ & 0.83 \\
SCD-Q c) (YES/NO) & $48 / 1$ & $44 / 5$ & 0.21 \\
SCD-Q score (/24) & $15.5 \pm 4.5$ & $14.7 \pm 5.7$ & 0.41 \\
Employed/unemployed & $39 / 10$ & $40 / 9$ & 1.00 \\
\hline
\end{tabular}

Data represent mean \pm SD. $p$-values were calculated by unpaired $t$ test for age, MMSE score, and SCD-Q score. $p$-values for male/female, SCD-Q a)-c), and employed/unemployed were calculated using $\chi^{2}$ test. MHBA, matured hop bitter acids; MMSE, Mini-Mental State Evaluation; SCD-Q, subjective cognitive decline questionnaire.

no significant differences in ingestion compliance between placebo or MHBA groups ( $>95.3 \%$ compliance). No serious adverse effects were observed in either group.

\section{Primary outcomes}

\section{Neuropsychological tests (attention)}

The primary endpoints of attention at the baseline and week twelve are summarized in Table 3. The week twelve of SDMT scores were signifi- cantly higher in the MHBA group compared to the placebo group ( $p=0.045)$ (Fig. 2). When comparing the baseline (week zero) and week twelve CAT neuropsychological tests, we observed a significant decrease in time to accomplish the task for both the Visual Cancellation test and the Position response test. We also observed higher SDMT scores in both groups, with a greater increase in the MHBA group. There were no significant differences in $\mathrm{z}$ score that integrated attention scores ( $\mathrm{z}$ score difference $=0.08$; $p=0.48$ at week twelve and $=0.15 ; p=0.18$ in the change from baseline to week twelve).

\section{Neuropsychological tests (memory)}

The primary memory endpoints at the baseline and week twelve are presented in Table 4. T6-T5 scores are not reported for two subjects (subject 1 in both groups) because they did not follow the rules of the test. Week twelve S-PA and TIM scores significantly increased in both groups when compared with their baseline scores. The week twelve T6-T5 scores for the RAVLT were significantly higher than the baseline scores in the placebo group. The TIM scores at week twelve were higher in the placebo group than in the MHBA group $(p=0.084)$. There were no significant differences in $\mathrm{z}$ score that integrated memory scores ( $\mathrm{z}$ score difference $=-0.17 ; p=0.25$ at week

Table 3

Mean scores for attention performance

\begin{tabular}{|c|c|c|c|c|c|}
\hline \multirow{2}{*}{\multicolumn{2}{|c|}{ Group }} & \multicolumn{2}{|c|}{ Baseline } & \multicolumn{2}{|c|}{ Week 12} \\
\hline & & mean \pm SD & $p$ & mean \pm SD & $p$ \\
\hline \multicolumn{6}{|c|}{ SDMT (achievement rate [\%]) } \\
\hline & Placebo & $49.9 \pm 6.1$ & 0.23 & $51.6 \pm 7.0^{*}$ & 0.045 \\
\hline & MHBA & $51.6 \pm 7.5$ & & $54.7 \pm 8.0^{* * \#}$ & \\
\hline \multicolumn{6}{|c|}{ Visual cancellation (time to accomplish the task [s]) } \\
\hline \multirow[t]{2}{*}{ Sign 1} & Placebo & $55.9 \pm 12.5$ & 0.51 & $48.8 \pm 8.4^{* *}$ & 0.67 \\
\hline & MHBA & $57.7 \pm 14.4$ & & $48.1 \pm 6.9^{* *}$ & \\
\hline \multirow[t]{2}{*}{ Sign 2} & Placebo & $60.6 \pm 12.4$ & 0.83 & $53.3 \pm 10.1^{* *}$ & 0.53 \\
\hline & MHBA & $61.1 \pm 10.4$ & & $52.1 \pm 8.2^{* *}$ & \\
\hline \multirow[t]{2}{*}{ Number "3" } & Placebo & $94.6 \pm 15.7$ & 0.91 & $86.0 \pm 12.8^{* *}$ & 0.43 \\
\hline & MHBA & $94.2 \pm 15.7$ & & $84.0 \pm 12.9^{* *}$ & \\
\hline \multirow[t]{2}{*}{ Japanese "ka" } & Placebo & $113.0 \pm 16.6$ & 0.97 & $104.0 \pm 15.0^{* *}$ & 0.65 \\
\hline & MHBA & $113.1 \pm 17.4$ & & $102.6 \pm 16.6^{* *}$ & \\
\hline \multicolumn{6}{|c|}{ Position response test (time to accomplish the task $[\mathrm{s}]$ ) } \\
\hline \multirow[t]{2}{*}{$1 \mathrm{st}$} & Placebo & $75.2 \pm 14.6$ & 0.56 & $68.5 \pm 10.8^{* *}$ & 0.70 \\
\hline & MHBA & $76.9 \pm 14.1$ & & $67.7 \pm 9.8^{* *}$ & \\
\hline \multirow[t]{2}{*}{ 2nd } & Placebo & $71.0 \pm 19.7$ & 0.99 & $65.3 \pm 11.0^{* *}$ & 0.91 \\
\hline & MHBA & $71.1 \pm 14.1$ & & $65.1 \pm 10.1^{* *}$ & \\
\hline \multicolumn{6}{|c|}{ Memory updating (achievement rate [\%]) } \\
\hline \multirow[t]{2}{*}{3 span } & Placebo & $83.7 \pm 15.7$ & 0.39 & $85.8 \pm 14.1$ & 0.59 \\
\hline & MHBA & $81.1 \pm 13.2$ & & $84.3 \pm 13.9$ & \\
\hline \multirow[t]{2}{*}{4 span } & Placebo & $68.4 \pm 21.9$ & 0.54 & $70.9 \pm 21.0$ & 0.59 \\
\hline & MHBA & $65.7 \pm 21.0$ & & $68.8 \pm 19.0$ & \\
\hline
\end{tabular}

Data represent mean \pm SD. $p$-values from unpaired $t$ test; (Placebo versus MHBA) are presented. MHBA group $(n=49)$; Placebo group $(n=49) ;{ }^{*} p<0.05 ;{ }^{* *} p<0.01$ (paired $t$ test compared with baseline); ${ }^{\#} p<0.05$ (unpaired $t$ test; Placebo versus MHBA). MHBA, matured hop bitter acids; SDMT, Symbol Digit Modalities Test. 


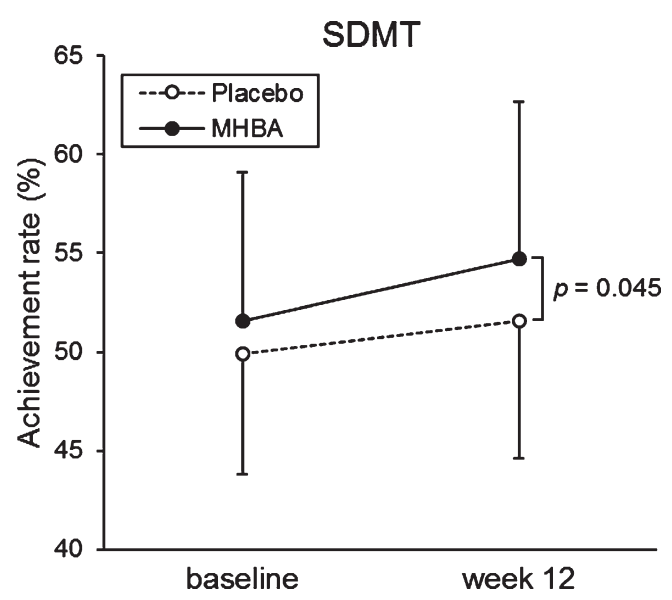

Fig. 2. The mean values of SDMT at baseline and week twelve. The solid line indicates the MHBA group $(n=49)$ and the dotted line indicates the placebo group $(n=49)$. Data points represent means and error bars indicate SD. $p$-value shows between-group differences performed using unpaired $t$ tests.

twelve and $=-0.09 ; p=0.48$ in the change from baseline to week twelve).

\section{Stress conditions}

To evaluate the level of stress, cortisol, $\mathrm{CgA}$, $\alpha$-amylase, and $\beta$-endorphin levels in saliva were measured at the baseline and week twelve and analyzed (Supplementary Table 1). Enough saliva for ELISA could not be collected from three subjects in the placebo group, therefore the following data were missing: three subjects for $\beta$-endorphin (before neuropsychological tests), one subject for $\beta$-endorphin (after neuropsychological tests), two subjects for cor- tisol (before neuropsychological tests), one subject for $\mathrm{CgA}$, and one subject for $\alpha$-amylase (before neuropsychological tests). Changes in $\beta$-endorphine levels from baseline to week twelve after neuropsychological tests were significantly lower in the MHBA group than in the placebo group ( $p=0.043)$ (Fig. 3). Furthermore, in the MHBA group, cortisol levels after neuropsychological tests and amylase levels before neuropsychological tests were significantly decreased at week twelve compared with levels at the baseline. There were no significant changes in cortisol and amylase levels observed in the placebo group.

\section{Subjective psychological assessment}

The change in score from baseline to week twelve in the "Anxiety" sub-category of the MIA-Q was tend to be lower in the MHBA group than the placebo group ( $p=0.096$, Supplementary Table 2, Fig. 4). In the "Task" sub-category, week twelve scores were significantly higher than the baseline scores in the MHBA group $(p=0.005)$, but not in the placebo group.

Week twelve anxiety-state STAI scores (after neuropsychological tests) significantly decreased in both groups compared to their baseline scores, but there was no difference in scores between the MHBA and placebo groups (Supplementary Table 3).

\section{Indicators in serum}

The results of indicators in serum are presented in Supplementary Table 4. The level of TTR at week twelve was significantly higher in the MHBA group than the placebo group $(p=0.048)$.

Table 4

Mean scores for memory performance

\begin{tabular}{|c|c|c|c|c|c|}
\hline & \multirow[t]{2}{*}{ Group } & \multicolumn{2}{|c|}{ Baseline } & \multicolumn{2}{|c|}{ Week 12} \\
\hline & & mean \pm SD & $p$ & mean \pm SD & $p$ \\
\hline \multicolumn{6}{|l|}{ S-PA } \\
\hline Unrelated pairs & $\begin{array}{l}\text { Placebo } \\
\text { MHBA }\end{array}$ & $\begin{array}{l}12.0 \pm 6.6 \\
10.7 \pm 6.5\end{array}$ & 0.33 & $\begin{array}{l}16.5 \pm 7.2^{* *} \\
15.2 \pm 8.4^{* *}\end{array}$ & 0.43 \\
\hline \multicolumn{6}{|l|}{ RAVLT } \\
\hline TIM & $\begin{array}{l}\text { Placebo } \\
\text { MHBA }\end{array}$ & $\begin{array}{c}42.0 \pm 7.9 \\
40.0 \pm 11.0\end{array}$ & 0.30 & $\begin{array}{c}46.1 \pm 8.6^{* *} \\
42.6 \pm 11.0^{* *}\end{array}$ & 0.084 \\
\hline T6-T5 & $\begin{array}{l}\text { Placebo } \\
\text { MHBA }\end{array}$ & $\begin{array}{l}-2.3 \pm 2.4 \\
-2.5 \pm 2.0\end{array}$ & 0.55 & $\begin{array}{c}-1.4 \pm 1.9^{*} \\
-1.9 \pm 2.3\end{array}$ & 0.24 \\
\hline $\mathrm{T} 7-\mathrm{T} 6$ & $\begin{array}{l}\text { Placebo } \\
\text { MHBA }\end{array}$ & $\begin{array}{l}-0.2 \pm 2.1 \\
-0.4 \pm 2.1\end{array}$ & 0.63 & $\begin{array}{l}-0.6 \pm 1.9 \\
-0.2 \pm 2.2\end{array}$ & 0.28 \\
\hline \multicolumn{6}{|l|}{ WMS-R } \\
\hline Figural memory & $\begin{array}{l}\text { Placebo } \\
\text { MHBA }\end{array}$ & $\begin{array}{l}7.0 \pm 1.6 \\
7.1 \pm 1.5\end{array}$ & 0.90 & $\begin{array}{l}7.4 \pm 1.2 \\
7.4 \pm 1.5\end{array}$ & 0.94 \\
\hline
\end{tabular}

Data represent mean \pm SD. $p$-value from unpaired $t$ test; (Placebo versus MHBA) are presented. MHBA group $(n=49)$; Placebo group $(n=49) ;{ }^{*} p<0.05,{ }^{* *} p<0.01$ (paired $t$ test compared with baseline). T6-T5 score were missing for two subjects (MHBA: 1 and Placebo: 1, respectively). MHBA, matured hop bitter acids; S-PA, Standard verbal paired-associate learning test; WMS-R, Wechsler Memory ScaleRevised; RAVLT, Rey Auditory Verbal Learning Test; TIM, Total Immediate Memory. 


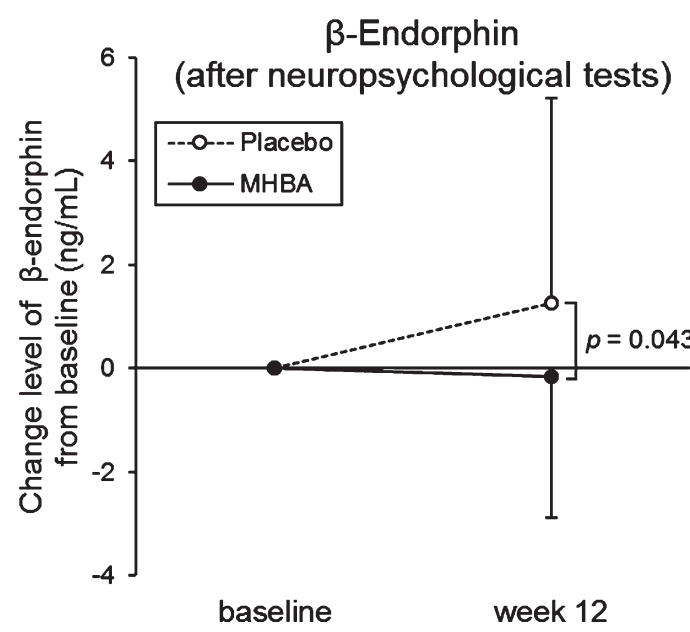

Fig. 3. Changes in $\beta$-Endorphin levels at baseline and week twelve (after the neuropsychological tests). The solid line indicates the MHBA group $(n=49)$ and the dotted line indicates the placebo group $(n=48)$. Data points represent means and error bars indicate SD. $p$-value shows between-group differences performed using unpaired $t$ tests.

\section{Subgroup analysis classified by the $S C D-Q$}

The characteristics of the SCD-C and SCD-P subgroups are presented in Supplementary Table 5. In the SCD-P subgroup, the SDMT score at week twelve was significantly higher in the MHBA group compared to the placebo group ( $p=0.011)$ (Fig. 5A). Furthermore, changes in the S-PA scores from the baseline to week twelve were significantly greater in the MHBA group compared to the placebo group $(p=0.033)$ (Fig. 5B). The MHBA group also remembered significantly fewer words (T7-T6) than the placebo group at week twelve compared to the baseline $(p=0.048)$ (Fig. 5C). Z score that integrated attention and memory scores tended to be higher in MHBA group than placebo group (z score difference $=0.21 ; p=0.11$ at week twelve and $=0.26 ; p=0.078$ in the change from baseline to week twelve). In the SCD-C subgroup, there were no significant differences between the MHBA and placebo groups in the neuropsychological tests.

\section{DISCUSSION}

In this randomized double-blind placebocontrolled trial, we evaluated the effects of twelve weeks of MHBA supplementation ( $35 \mathrm{mg} /$ day) in healthy older adults. We observed improved attention (assessed by the SDMT) and reduced stress after neuropsychological tests (assessed by

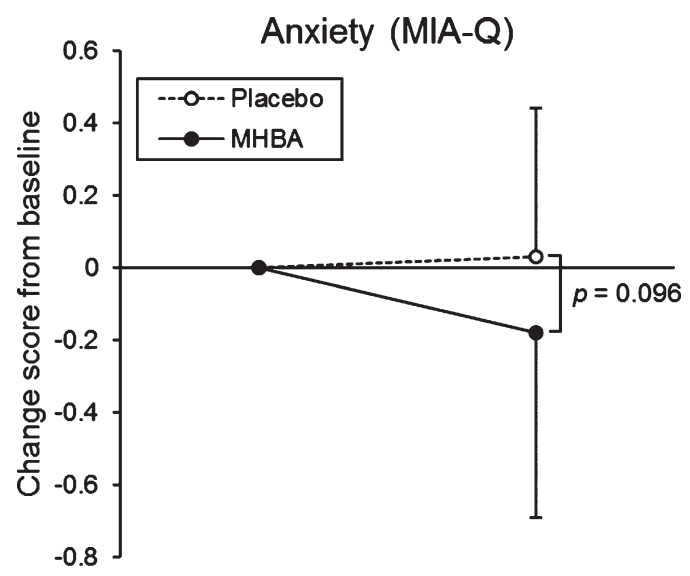

baseline week 12

Fig. 4. Changes in anxiety score in the MIA-Q at baseline and week twelve. The solid line indicates the MHBA group $(n=49)$ and the dotted line indicates the placebo group $(n=49)$. Data points represent means and error bars indicate SD. $p$-value shows between-group differences performed using Mann-Whitney $U$ tests.

$\beta$-endorphin) in the participants who received MHBA supplements.

SDMT performance is an extremely sensitive predictor of progression from amnestic MCI to dementia [35]. The SDMT assesses mental processing speed and concentration functions, in addition to divided attention [36]. Previous imaging studies reported that cognitive operations in multiple brain areas including the bilateral middle frontal, inferior frontal and lingual gyri, superior parietal lobule, declive, cuneus, precuneus, and dorsolateral prefrontal cortex (dlPFC) are highly involved in the performance of the SDMT $[37,38]$. Our results are consistent with reports from a previous clinical trial that MHBA improved verbal fluency and Stroop test (color-word interference) performance, both of which reflect dlPFC function [15].

Previous studies also report that VNS therapy improved SDMT performance in refractory depression patients [39]. Additionally, locus-noradrenergic systems are responsible for cognitive processes including attention [40], and methylphenidate, a stimulant drug with dopamine and norepinephrine reuptake inhibition properties, improves attention in healthy volunteers [41]. Thus, the underlying mechanism for improved attention in our MHBA group may be increased norepinephrine levels via MHBAinduced VNS, as suggested by preclinical studies.

MHBA supplementation reduced the levels of $\beta$-endorphin after the neuropsychological tests. 


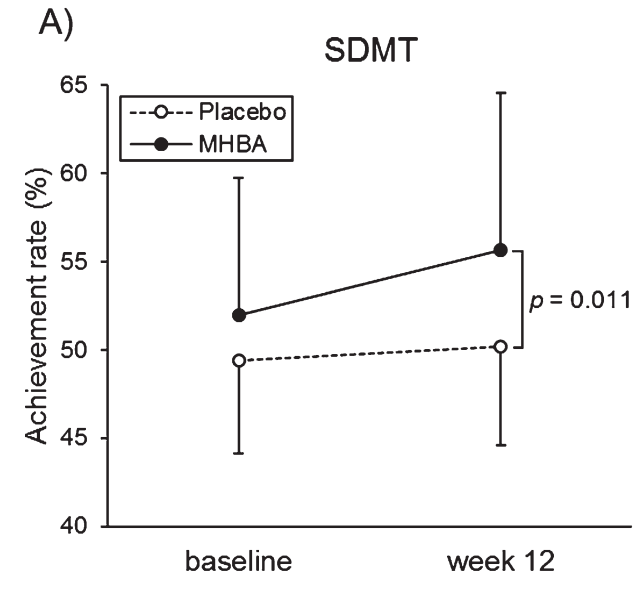

B)
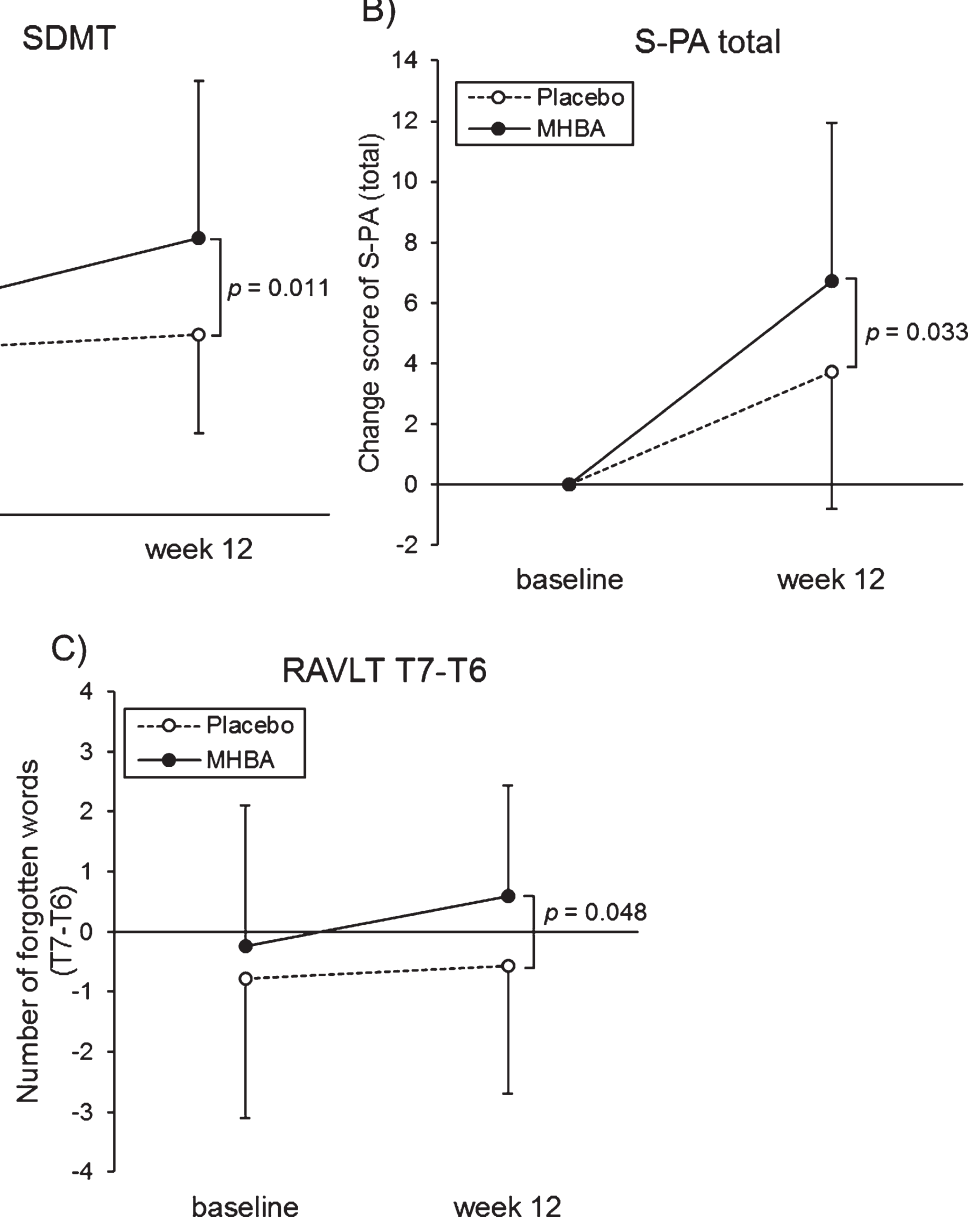

Fig. 5. A) The mean values of SDMT at baseline and week twelve, B) changes in unrelated words scores of the S-PA from baseline, C) the mean values of T7-T6 in the RAVLT at baseline and week twelve in the SCD-P. The solid line indicates the MHBA group ( $n=22)$ and the dotted line indicates the placebo group $(n=28)$. Data points represent means and error bars indicate SD. $p$-values show between-group differences performed using unpaired $t$ tests.

$\beta$-Endorphin is a marker of hypothalamic-pituitaryadrenal (HPA)-axis activity [42], which is used to evaluate psychological stress (e.g., stress caused by public speaking, watching stressful videos, or taking examinations) [43-45]. Additionally, patients suffering from depression are reported to have higher $\beta$-endorphin levels [46, 47]. Cortisol, another marker for stress reactions caused by the HPA-axis [48], and $\beta$-endorphin levels were significantly lower after neuropsychological tests in subjects who received the MHBA supplement (Supplementary Table 1). $\alpha$-Amylase and $\mathrm{CgA}$ reflect sympathetic-adrenalmedullary (SAM)-axis response to acute stress [49, 50]. $\alpha$-amylase and $\mathrm{CgA}$ levels were not affected by the MHBA supplementation. This could be because the stress markers regulated by the SAMaxis decrease back to the baseline faster than those regulated by the HPA-axis [51]. Saliva was collected 15 minutes before and after the neuropsychological test, therefore our study design did not detect any effect of MHBA on stress response using SAM-axis markers. In future studies, to evaluate the effect of MHBA on mental stress in more detail, it would be necessary to set more frequent saliva collection time points.

The Anxiety sub-category of the MIA-Q [29], which evaluated both the introspective knowledge of one's own memory capabilities (and strategies that can aid memory) and the processes involved in memory self-monitoring, tended to improve in the MHBA group compared with the placebo group. This result was consistent with our previous study in which daily anxiety-state (evaluated by questionnaire) was improved by MHBA supplementation [15]. 
Anxiety-trait was a robust indicator of the subject's personality and changed little before and after the intervention. Anxiety-state, which reflects transient anxiety to the neuropsychological tests, and autonomic nerve activity were assessed before and after the neuropsychological tests because anxiety-state may affect the neuropsychological test performance [30-32]. We confirmed that there were no significant differences between the groups.

In the present study, putative MCI markers were evaluated and the level of TTR, which acts as an A $\beta$ protein scavenger, was significantly higher at week twelve in the MHBA group than in the placebo group $(p=0.048)$ (Supplementary Table 4$)[16]$. A previous study by our research group reported supplementation of IAAs, the bitter component derived from hops, induced TTR expression in AD model mice [52]. Thus, the elevated TTR levels observed in the present study indicate that MHBA supplementation may be a viable $\mathrm{AD}$ therapy, but longer trials must be considered.

Furthermore, previous study indicated that MHBA improved the energy metabolism in over weighted subjects [53]. Since obesity is known risk factor for dementia [54], MHBA may prevent dementia by improving metabolic function. In the future study, we will design the clinical trial to evaluate the effect of MHBA on the association of energy metabolism with cognition.

Previous studies reported that SCD groups were heterogeneous (i.e., memory complaints were associated with physical health problems, depression, feelings of underachievement, low perceived selfefficacy, and high neuroticism). In particular, it has been reported that the characteristics of subjects differ depending on whether studies on SCD were recruited from community volunteers or clinical patients [55]. Community-based SCD subjects who perceived selfcognitive decline but did not seek medical help were classified as SCD-P and clinic-based SCD subjects who sought medical help were classified as SCD-C. In the current study, SCD-C and SCD-P were classified by three questions in the SCD questionnaire [17, 18]. Previous reports indicate that SCD-P classification suggests that $\mathrm{AD}-$ like pathology is less advanced than SCD-C. For example, hippocampal atrophy was specifically associated with medical help-seeking participants (SCD-C) [55]. Our subgroup analysis revealed that SDMT and memory retrieval scores were improved by the intake of MHBA in the SCD-P group. Thus, we hypothesize that early intervention with MHBA supplementation (before the onset of hippocampal atrophy) could be more beneficial for the improvement of brain function, but further study based on functional MRI is required.

We acknowledge the limitations of this study. First, as the result of performing the analysis considering multiplicity by the integrated analysis using z scores, there were no significant differences between the groups. In the future, it is necessary to verify the effect of MHBA on cognitive function from multiple study results by meta-analysis. Furthermore, we could not evaluate whether MHBA supplementation prevented age-related cognitive decline due to the relatively short study length. Future long-term studies would be required to assess the effects of MHBA supplementation on aging-related changes in order to avoid the learning and ceiling effects caused by repetition of the same test. Additionally, the mechanism underlying the cognition-improving effects of MHBA via vagus nerve has not been confirmed, therefore further study is required to elucidate the mechanism.

In conclusion, the present study results showed that MHBA supplementation improved mental processing speed, attention, and concentration and reduced mental stress after intellectual work in healthy adults aged 45-69 years with SCD. In particular, early intervention through MHBA supplementation in persons with SCD could be successful in improving cognitive function.

\section{ACKNOWLEDGMENTS}

We thank Mr. Yuji Kaneko for preparing the test tablets used in this experiment.

This study was funded by the Kirin Holdings Company, Limited. The funder designed this study and supplied the tests capsules and placebo. The funder, however, had no role in data collection or analysis. The manuscript was prepared by members of the funder and the funder decided to publish the results.

Authors' disclosures available online (https:// www.j-alz.com/manuscript-disclosures/20-0229r1).

\section{SUPPLEMENTARY MATERIAL}

The supplementary material is available in the electronic version of this article: https://dx.doi.org/ 10.3233/JAD-200229.

\section{REFERENCES}

[1] Barnard ND, Bush AI, Ceccarelli A, Cooper J, de Jager CA, Erickson KI, Fraser G, Kesler S, Levin SM, Lucey B, Morris 
MC, Squitti R (2014) Dietary and lifestyle guidelines for the prevention of Alzheimer's disease. Neurobiol Aging 35 Suppl 2, S74-78.

[2] Rabin LA, Smart CM, Amariglio RE (2017) Subjective cognitive decline in preclinical Alzheimer's disease. Annu Rev Clin Psychol 13, 369-396.

[3] Merrill CA, Jonsson MA, Minthon L, Ejnell H, H CsS, Blennow K, Karlsson M, Nordlund A, Rolstad S, Warkentin S, Ben-Menachem E, Sjogren MJ (2006) Vagus nerve stimulation in patients with Alzheimer's disease: Additional follow-up results of a pilot study through 1 year. J Clin Psychiatry 67, 1171-1178.

[4] Sjogren MJ, Hellstrom PT, Jonsson MA, Runnerstam M, Silander HC, Ben-Menachem E (2002) Cognitionenhancing effect of vagus nerve stimulation in patients with Alzheimer's disease: A pilot study. J Clin Psychiatry 63, 972-980.

[5] Johnson RL, Wilson CG (2018) A review of vagus nerve stimulation as a therapeutic intervention. J Inflamm Res 11, 203-213.

[6] Horvat P, Richards M, Kubinova R, Pajak A, Malyutina S, Shishkin S, Pikhart H, Peasey A, Marmot MG, SinghManoux A, Bobak M (2015) Alcohol consumption, drinking patterns, and cognitive function in older Eastern European adults. Neurology 84, 287-295.

[7] Neafsey EJ, Collins MA (2011) Moderate alcohol consumption and cognitive risk. Neuropsychiatr Dis Treat 7, 465-484

[8] Kennedy DO, Wightman EL, Reay JL, Lietz G, Okello EJ, Wilde A, Haskell CF (2010) Effects of resveratrol on cerebral blood flow variables and cognitive performance in humans: A double-blind, placebo-controlled, crossover investigation. Am J Clin Nutr 91, 1590-1597.

[9] Ano Y, Dohata A, Taniguchi Y, Hoshi A, Uchida K, Takashima A, Nakayama H (2017) Iso-alpha-acids, bitter components of beer, prevent inflammation and cognitive decline induced in a mouse model of Alzheimer's disease. J Biol Chem 292, 3720-3728.

[10] Ano Y, Ohya R, Kondo K, Nakayama H (2019) Iso-alphaacids, hop-derived bitter components of beer, attenuate age-related inflammation and cognitive decline. Front Aging Neurosci 11, 16.

[11] Ayabe T, Ohya R, Taniguchi Y, Shindo K, Kondo K, Ano Y (2018) Matured hop-derived bitter components in beer improve hippocampus-dependent memory through activation of the vagus nerve. Sci Rep 8, 15372 .

[12] Fukuda T, Ohya R, Kobayashi K, Ano Y (2019) Matured hop bitter acids in beer improve lipopolysaccharide-induced depression-like behavior. Front Neurosci 13, 41.

[13] Taniguchi Y, Matsukura Y, Ozaki H, Nishimura K, Shindo $\mathrm{K}$ (2013) Identification and quantification of the oxidation products derived from alpha-acids and beta-acids during storage of hops (Humulus lupulus L.). J Agric Food Chem 61, 3121-3130.

[14] Taniguchi Y, Matsukura Y, Taniguchi H, Koizumi H, Katayama M (2015) Development of preparative and analytical methods of the hop bitter acid oxide fraction and chemical properties of its components. Biosci Biotechnol Biochem 79, 1684-1694.

[15] Fukuda T, Obara K, Saito J, Umeda S, Ano Y (2020) Effects of hop bitter acids, bitter components in beer, on cognition in healthy adults: A randomized controlled trial. J Agric Food Chem 68, 206-212.

[16] Uchida K, Shan L, Suzuki H, Tabuse Y, Nishimura Y, Hirokawa Y, Mizukami K, Akatsu H, Meno K, Asada
T (2015) Amyloid-beta sequester proteins as blood-based biomarkers of cognitive decline. Alzheimers Dement (Amst) 1, 270-280.

[17] Rami L, Mollica MA, Garcia-Sanchez C, Saldana J, Sanchez B, Sala I, Valls-Pedret C, Castellvi M, Olives J, Molinuevo JL (2014) The Subjective Cognitive Decline Questionnaire (SCD-Q): A validation study. J Alzheimers Dis 41, 453-466.

[18] Hiroyuki K (2018) Kenzai-hasshomae-shindan-jidai-nomonowasure-gairai-jushinsha-toha (Japanese). Jpn J Geriatr Psychiatry 29, 486-492.

[19] Taniguchi Y, Yamada M, Taniguchi H, Matsukura Y, Shindo K (2015) Chemical characterization of beer aging products derived from hard resin components in hops (Humulus lupulus L.). J Agric Food Chem 63, 10181-10191.

[20] Taniguchi Y, Taniguchi H, Matsukura Y, Kawachi Y, Shindo K (2014) Structural elucidation of humulone autoxidation products and analysis of their occurrence in stored hops. $J$ Nat Prod 77, 1252-1261.

[21] Burns NR, Bryan J, Nettelbeck T (2006) Ginkgo biloba: No robust effect on cognitive abilities or mood in healthy young or older adults. Hum Psychopharmacol 21, 27-37.

[22] Jackson PA, Forster JS, Bell JG, Dick JR, Younger I, Kennedy DO (2016) DHA supplementation alone or in combination with other nutrients does not modulate cerebral hemodynamics or cognitive function in healthy older adults. Nutrients 8, 86.

[23] Stough C, Downey L, Silber B, Lloyd J, Kure C, Wesnes K, Camfield D (2012) The effects of 90-day supplementation with the omega-3 essential fatty acid docosahexaenoic acid (DHA) on cognitive function and visual acuity in a healthy aging population. Neurobiol Aging 33, 824 e821-823.

[24] Kato M (2006) The development and standardization of Clinical Assessment for Attention (CAT) and Clinical Assessment for Spontaneity (CAS) (Japanese). Higher Brain Function Res 26, 310-319.

[25] Rey A (1964) The Clinical Examination in Psychology. Presses Universitaires de France, Paris.

[26] Japan Society for Higher Brain Dysfunction (2011) Standard Verbal Paired-Associate Learning Test. Shinkohigakushunppansha, Tokyo.

[27] Koike A, Sugishita M (2011) The Japanese version of the Wechsler memory scale-revised. Nihon Rinsho 69, 408-412.

[28] Spielberger CD, Sydeman SJ (1994) State-Trait Anxiety Inventory and State-Trait Anger Expression Inventory. In The Use of Psychological Testing for Treatment Planning and Outcome Assessment, Maruish ME, ed. Lawrence Erlbaum Associates, Inc., pp. 292-321.

[29] Kinjo H, Ide S, Ishihara O (2013) Structure of the Japanese Metamemory in Adulthood (MIA) questionnaire and development of its abridged version. Ninchi Shinrigaku Kenkyu 11, 31-41.

[30] Culler RE, Holahan CJ (1980) Test anxiety and academic performance: The effects of study-related behaviors. JEduc Psychol 72, 16-20.

[31] Jerrell CC, Ronald E J (2002) Cognitive test anxiety and academic performance. Contemp Educ Psychol 27, 270-295.

[32] Kleijn WC, van der Ploeg HM, Topman RM (1994) Cognition, study habits, test anxiety, and academic performance. Psychol Rep 75, 1219-1226.

[33] Akerstedt T, Gillberg M (1990) Subjective and objective sleepiness in the active individual. Int J Neurosci 52, 29-37.

[34] Valech N, Tort-Merino A, Coll-Padros N, Olives J, Leon M, Rami L, Molinuevo JL (2018) Executive and language subjective cognitive decline complaints discriminate preclinical 
Alzheimer's disease from normal aging. $J$ Alzheimers Dis 61, 689-703.

[35] Fleisher AS, Sowell BB, Taylor C, Gamst AC, Petersen RC, Thal LJ (2007) Clinical predictors of progression to Alzheimer disease in amnestic mild cognitive impairment. Neurology 68, 1588-1595.

[36] Lopez OL, Mackell JA, Sun Y, Kassalow LM, Xu Y, McRae $\mathrm{T}, \mathrm{Li} \mathrm{H}$ (2008) Effectiveness and safety of donepezil in Hispanic patients with Alzheimer's disease: A 12-week open-label study. J Natl Med Assoc 100, 1350-1358.

[37] Luan Y, Wang C, Jiao Y, Tang T, Zhang J, Teng GJ (2019) Prefrontal-temporal pathway mediates the cross-modal and cognitive reorganization in sensorineural hearing loss with or without tinnitus: A multimodal MRI study. Front Neurosci $13,222$.

[38] Silva PHR, Spedo CT, Barreira AA, Leoni RF (2018) Symbol Digit Modalities Test adaptation for magnetic resonance imaging environment: A systematic review and meta-analysis. Mult Scler Relat Disord 20, 136-143.

[39] Desbeaumes Jodoin V, Richer F, Miron JP, FournierGosselin MP, Lesperance P (2018) Long-term sustained cognitive benefits of vagus nerve stimulation in refractory depression. J ECT 34, 283-290.

[40] Berridge CW, Waterhouse BD (2003) The locus coeruleusnoradrenergic system: Modulation of behavioral state and state-dependent cognitive processes. Brain Res Brain Res $\operatorname{Rev}$ 42, 33-84

[41] Linssen AM, Sambeth A, Vuurman EF, Riedel WJ (2014) Cognitive effects of methylphenidate in healthy volunteers: A review of single dose studies. Int J Neuropsychopharmacol 17, 961-977.

[42] Charmandari E, Tsigos C, Chrousos G (2005) Endocrinology of the stress response. Annu Rev Physiol 67, 259-284.

[43] Kyaimon M, R J, See-Ziau H, MS K, Sau-Kuen L (2017) Cortisol, $\beta$-endorphin and oxidative stress markers in healthy medical students in response to examination stress. Biomed Res 28, 3774-3779.

[44] Sheps DS, Ballenger MN, De Gent GE, Krittayaphong R, Dittman E, Maixner W, McCartney W, Golden RN, Koch G, Light KC (1995) Psychophysical responses to a speech stressor: Correlation of plasma beta-endorphin levels at rest and after psychological stress with thermally measured pain threshold in patients with coronary artery disease. J Am Coll Cardiol 25, 1499-1503.

[45] Takai N, Yamaguchi M, Aragaki T, Eto K, Uchihashi K, Nishikawa Y (2007) Gender-specific differences in salivary biomarker responses to acute psychological stress. Ann NY Acad Sci 1098, 510-515.
[46] Darko DF, Risch SC, Gillin JC, Golshan S (1992) Association of beta-endorphin with specific clinical symptoms of depression. Am J Psychiatry 149, 1162-1167.

[47] Goodwin GM, Austin MP, Curran SM, Ross M, Murray C, Prentice N, Ebmeier KP, Bennie J, Carroll S, Dick H, Fink G (1993) The elevation of plasma beta-endorphin levels in major depression. J Affect Disord 29, 281-289.

[48] Poll EM, Kreitschmann-Andermahr I, Langejuergen Y, Stanzel S, Gilsbach JM, Gressner A, Yagmur E (2007) Saliva collection method affects predictability of serum cortisol. Clin Chim Acta 382, 15-19.

[49] Florio P, Mezzesimi A, Turchetti V, Severi FM, Ticconi C, Forconi S, Petraglia F (2002) High levels of human chromogranin A in umbilical cord plasma and amniotic fluid at parturition. J Soc Gynecol Investig 9, 32-36.

[50] Nater UM, La Marca R, Florin L, Moses A, Langhans W, Koller MM, Ehlert U (2006) Stress-induced changes in human salivary alpha-amylase activity — associations with adrenergic activity. Psychoneuroendocrinology 31, 49-58.

[51] Ohara K, Misaizu A, Kaneko Y, Fukuda T, Miyake M, Miura Y, Okamura H, Yajima J, Tsuda A (2018) beta-Eudesmol, an oxygenized sesquiterpene, reduces the increase in saliva 3-Methoxy-4-Hydroxyphenylglycol after the "Trier Social Stress Test" in healthy humans: A randomized, doubleblind, placebo-controlled cross-over study. Nutrients 11, E9.

[52] Fukuda T, Uchida K, Nakayama H, Ano Y (2018) Short-term administration of iso-alpha-acids increases transthyretin transcription in the hippocampus. Biochem Biophys Res Commun 507, 471-475.

[53] Morimoto-Kobayashi Y, Ohara K, Ashigai H, Kanaya T, Koizumi K, Manabe F, Kaneko Y, Taniguchi Y, Katayama M, Kowatari Y, Kondo S (2016) Matured hop extract reduces body fat in healthy overweight humans: A randomized, double-blind, placebo-controlled parallel group study. $\mathrm{Nutr}$ $J 15,25$.

[54] Livingston G, Sommerlad A, Orgeta V, Costafreda SG, Huntley J, Ames D, Ballard C, Banerjee S, Burns A, CohenMansfield J, Cooper C, Fox N, Gitlin LN, Howard R, Kales HC, Larson EB, Ritchie K, Rockwood K, Sampson EL, Samus Q, Schneider LS, Selbaek G, Teri L, Mukadam N (2017) Dementia prevention, intervention, and care. Lancet 390, 2673-2734.

[55] Perrotin A, La Joie R, de La Sayette V, Barre L, Mezenge F, Mutlu J, Guilloteau D, Egret S, Eustache F, Chetelat G (2017) Subjective cognitive decline in cognitively normal elders from the community or from a memory clinic: Differential affective and imaging correlates. Alzheimers Dement 13, 550-560. 